\title{
Usefulness of computational fluid dynamics in penetrating aortic ulcer
}

\author{
Ferdinando Auricchio ${ }^{1}$, Michele Conti ${ }^{1}$, Rodrigo M. Romarowski ${ }^{2}$ \\ ${ }^{1}$ Department of Civil Engineering and Architecture, University of Pavia, Pavia, Italy; ${ }^{2} 3 \mathrm{D}$ and Computer Simulation Laboratory, IRCCS-Policlinico \\ San Donato, San Donato Milanese, Italy \\ Correspondence to: Ferdinando Auricchio. Department of Civil Engineering and Architecture, University of Pavia, Pavia, Italy. Email: auricchio@unipv.it.
}

Submitted Mar 16, 2019. Accepted for publication Jun 11, 2019.

doi: $10.21037 /$ acs.2019.06.08

View this article at: http://dx.doi.org/10.21037/acs.2019.06.08

Computational fluid dynamics (CFD) is a numerical technique aimed at solving a system of partial differential equations describing fluid motion in a complex $3 \mathrm{D}$ arbitrary domain (1). Ever since the first CFD models were developed, there has been immense interest not only in industrial applications but also in the simulation of blood flow.

Early hemodynamic simulations were run in idealized geometries resembling human vasculature and boundary conditions were mostly reproduced from physiology books. Two main contributions helped to leap forward and arrive where we are today: the improvement in imaging methods, which gave researchers the opportunity to create patient-specific models, including geometry and flow measurements, and the continuous increase in computer memory and processing power (2). With these two obstacles becoming increasingly more manageable, it is now possible to run a simplified simulation of blood flow on any personal computer if the timeframe for results is not critical. Authors focused their CFD efforts first in healthy aortas and then moved to diseases, such as aneurysms (3), dissections and penetrating aortic ulcers (PAU), trying to find the hemodynamic pathways that may predict the evolution of the patient by further stratifying risk in comparison with "classical" clinical indicators (i.e., aortic diameter) (4).

As an example, PAU is a disease characterized by two biomechanical components that closely interact: the fluid dynamics of blood, and the mechanical response of the damaged elastic lamina and the hematoma in the media layer. Such features make the mechanism of rupture different from a thoracic aortic aneurysm, where the aorta is dilated and sometimes intraluminal thrombus is present from a dissection (where a parallel cavity for blood is created in the media) (5).

It is now clear that the question we would like to answer with computational simulations is: which patient with a PAU is more likely to develop further damage to the different layers of the aortic wall, leading to growth of the hematoma or even rupture? To do so, CFD falls short in describing the blood-tissue interaction since simulations are typically done within a rigid wall (6). A more complete computational model is possible using fluid-structure interaction (FSI) as well as detailed modelling of the behaviour of the tissues that constitute each layer of the aortic wall (7). However, even if all these requisites were met, there would still be inter-individual variability in tissue characteristics that are impossible to acquire by means of non-invasive techniques in each diseased patient.

Even if this hypothetical blood-tissue interaction model did not include much uncertainty, there would still be a crucial step needed for taking simulations to clinical practice: validation against clinical outcomes. Fortunately, the incidence of PAU is low (as compared to other vascular diseases) and current guidelines give clear indications on when surgery (either endovascular or open) is indicated. Since the risk of treatment clearly outweighs the lifethreatening risk of bleeding after rupture, nobody would agree to test the validity of computational simulations by a wait-and-watch strategy for patients with increasing PAU diameter.

Most hemodynamic indicators retrieved from CFD that are currently available in literature [ranging from Wall Shear Stress and Displacement Forces (8) to more complex indicators (9)] have not yet demonstrated any 
utility in independently predicting the evolution of disease. Accordingly, there are still several challenging steps to execute reliable CFD models. First, creating a model able to predict the rupture behaviour of the combined aortic layers even without the effect of blood. Secondly, the computational model should include a FSI simulation framework, where the fluid can interact with the aortic wall in a realistic way. Finally (and importantly), the simulations should be "multiscale", not only in space, but also in time: simulations should cover what happens in each heartbeat as well as the long-term impact of pathologic hemodynamics in the aortic tissue.

In conclusion, advances in CFD have made simulations of blood flow in diseased aortic reconstructions increasingly accurate. However, the complexity of including bloodtissue interaction in a multiscale analysis limits the ability of researchers to predict the course of PAU.

\section{Acknowledgments}

None.

\section{Footnote}

Conflicts of Interest: The authors have no conflicts of interest to declare.

\section{References}

1. van Bogerijen GH, Tolenaar JL, Conti M, et al. Contemporary Role of Computational Analysis in Endovascular Treatment for Thoracic Aortic Disease.

Cite this article as: Auricchio F, Conti M, Romarowski RM. Usefulness of computational fluid dynamics in penetrating aortic ulcer. Ann Cardiothorac Surg 2019;8(4):492-493. doi: 10.21037/acs.2019.06.08
Aorta (Stamford) 2013;1:171-81.

2. Auricchio F, Conti M, Lefieux A, et al. Patient-specific analysis of post-operative aortic hemodynamics: a focus on thoracic endovascular repair (TEVAR). Comput Mech 2014;54:943-53.

3. Shipkowitz T, Rodgers VG, Frazin LJ, et al. Numerical study on the effect of secondary flow in the human aorta on local shear stresses in abdominal aortic branches. J Biomech 2000;33:717-28.

4. Biasetti J, Gasser TC, Auer M, et al. Hemodynamics of the normal aorta compared to fusiform and saccular abdominal aortic aneurysms with emphasis on a potential thrombus formation mechanism. Ann Biomed Eng 2010;38:380-90.

5. Hayashi H, Matsuoka Y, Sakamoto I, et al. Penetrating atherosclerotic ulcer of the aorta: imaging features and disease concept. Radiographics 2000;20:995-1005.

6. van Bakel TM, Romarowski RM, Morganti S, et al. Blood Flow after Endovascular Repair in the Aortic Arch: A Computational Analysis. Aorta (Stamford) 2018;6:81-7.

7. Xenos M, Labropoulos N, Rambhia S, et al. Progression of abdominal aortic aneurysm towards rupture: refining clinical risk assessment using a fully coupled fluid-structure interaction method. Ann Biomed Eng 2015;43:139-53.

8. Marrocco-Trischitta MM, Romarowski RM, de Beaufort HW, et al. The Modified Arch Landing Areas Nomenclature identifies hostile zones for endograft deployment: a confirmatory biomechanical study in patients treated by thoracic endovascular aortic repair. Eur J Cardiothorac Surg 2019;55:990-7.

9. Bianchi D, Monaldo E, Gizzi A, et al. A FSI computational framework for vascular physiopathology: A novel flowtissue multiscale strategy. Med Eng Phys 2017;47:25-37. 